\title{
Diagnostic Accuracy of High-Resolution Black-Blood MRI in the Evaluation of Intracranial Large-Vessel Arterial Occlusions
}

\author{
(D)A.S. Al-Smadi, (D).N. Abdalla, (D)A.H. Elmokadem, (D)A. Shaibani, (D) M.C. Hurley, (DM.B. Potts, DB.S. Jahromi, (D)T.J. Carroll, and \\ (iD).A. Ansari \\ $\mathrm{O} \equiv$
}

\begin{abstract}
BACKGROUND AND PURPOSE: 3D high-resolution black-blood MRI or MR vessel wall imaging allows evaluation of the intracranial arterial wall and extraluminal pathology. We investigated the diagnostic accuracy and reliability of black-blood MRI for the intraluminal detection of large-vessel arterial occlusions.
\end{abstract}

MATERIALS AND METHODS: We retrospectively identified patients with intracranial arterial occlusions, confirmed by CTA or DSA, who also underwent 3D black-blood MRI with nonenhanced and contrast-enhanced TI sampling perfection with application-optimized contrasts by using different flip angle evolution (TI SPACE) sequences. Black-blood MRI findings were evaluated by 2 independent and blinded neuroradiologists. Large-vessel intracranial arterial segments were graded on a 3-point scale (grades 0-2) for intraluminal baseline $\mathrm{T1}$ hyperintensity and contrast enhancement. Vessel segments were considered positive for arterial occlusion if focal weak (grade 1) or strong (grade 2) T1-hyperintense signal and/or enhancement replaced the normal intraluminal black-blood signal.

RESULTS: Thirty-one patients with 38 intracranial arterial occlusions were studied. The median time interval between black-blood MRI and CTA/DSA reference standard studies was 2 days (range, $0-20$ days). Interobserver agreement was good for $T 7$ hyperintensity $(\kappa=0.63$ ) and excellent for contrast enhancement $(\kappa=0.89)$. High sensitivity $(100 \%)$ and specificity $(99.8 \%)$ for intracranial arterial occlusion diagnosis was observed with either intraluminal $\mathrm{T} 1$ hyperintensity or contrast-enhancement imaging criteria on black-blood MRI. Strong grade 2 intraluminal enhancement was maintained in $>80 \%$ of occlusions irrespective of location or chronicity. Relatively increased strong grade 2 intraluminal $\mathrm{Tl}$ hyperintensity was noted in chronic/incidental versus acute/subacute occlusions (45.5\% versus $12.5 \%, P=.04)$.

CONCLUSIONS: Black-blood MRI with or without contrast has high diagnostic accuracy and reliability in evaluating intracranial largevessel arterial occlusions with near-equivalency to DSA and CTA.

ABBREVIATIONS: BBMRI = black-blood MRI; MR VWI = MR vessel wall imaging; SPACE = sampling perfection with application-optimized contrasts by using different flip angle evolution

A cute ischemic stroke secondary to intracranial large-vessel thromboembolic occlusion has substantial morbidity and mortality. Emergent intervention in large-vessel occlusions with

Received January 31, 2019; accepted after revision April 10.

From the Departments of Radiology (A.S.A.-S., R.N.A., A.H.E., A.S., M.C.H., M.B.P., B.S.J., S.A.A.), Neurology (S.A.A.), and Neurological Surgery (A.S., M.C.H., M.B.P., B.S.J., S.A.A.), Northwestern University, Feinberg School of Medicine, Chicago, Illinois; Department of Radiology (T.J.C.), University of Chicago, Chicago, Illinois; Department of Radiology (R.N.A.), Ain Shams University, Cairo, Egypt; and Department of Radiology (A.H.E.), Mansoura University, Mansoura, Egypt.

This work was supported by the following grants: American Heart Association 13GRNT17340018 and 14GRNT20380798 (Principal Investigators: S.A. Ansari and T.J. Carroll); National Institutes of Health/National Heart, Lung, and Blood Institute 1R21HL130969 (Principal Investigator: S.A. Ansari); and National Institutes of Health/ National Heart, Lung, and Blood Institute IR01NS093908 (Principal Investigator: T.J. Carroll).

Please address correspondence to Sameer A. Ansari, MD, PhD, Departments of Radiology, Neurology, and Neurological Surgery, Northwestern University, Feinberg School of Medicine, 676 N St Clair St, Suite 800, Chicago, IL 60611-2927;

e-mail: s-ansari@northwestern.edu intravenous thrombolysis and/or endovascular thrombectomy has been shown to dramatically improve clinical outcomes. ${ }^{1}$ However, even delayed diagnosis of complete intracranial arterial occlusions and differentiation from intracranial vessel wall pathologies impact medical and surgical management. Currently, neurovascular imaging is performed using various imaging modalities, including CTA, MRA, and DSA, but the limited assessment of the arterial wall cannot reliably differentiate in situ pathologies such as intracranial atherosclerosis, dissection, vasculitis, or steno-occlusive vasculopathy. Due to the superior contrast and high spatial resolution of 3D black-blood MR imaging (BBMRI) or MR vessel wall imaging (MR VWI), the extraluminal vessel wall can be clearly distinguished from the

\footnotetext{
- Indicates open access to non-subscribers at www.ajnr.org

$\equiv$ Indicates article with supplemental on-line table.

http://dx.doi.org/10.3174/ajnr.A6065
} 
intraluminal black-blood compartment, allowing improved diagnosis and delineation of intravascular thromboembolic occlusions and their related blood products. ${ }^{2}$

3D-TOF-MRA is considered the most common MRA technique for evaluating intracranial steno-occlusive disease in acute ischemic stroke, often as an adjunct to MR diffusion-weighted imaging. ${ }^{3,4}$ However, 3D-TOF-MRA has been shown to be insensitive due to poor flow enhancement from in-plane dephasing or slow-flow artifacts, ${ }^{5}$ leading to false-positive results either by upgrading the stenosis severity or a false diagnosis of a vascular occlusion. ${ }^{6}$ Although the T1 spin-echo sequence can accurately identify subacute hemorrhage or thrombus as signal hyperintensity on conventional MR imaging due to the presence of methemoglobin, ${ }^{7}$ it has major limitations in the detection of intra-arterial thrombosis, including lower resolution, flow artifacts from inadequate double inversion recovery flow-suppression techniques, and chronologic variance of signal intensity, depending on the stage of thrombus evolution (oxy-/deoxy-hemoglobin, intra-/extracellular methemoglobin, and hemosiderin). Conversely, BBMRI using the T1 sampling perfection with application-optimized contrasts by using different flip angle evolution (SPACE; Siemens, Erlangen, Germany) sequences with intravoxel phase dispersion provides excellent intravascular black-blood flow suppression with intravoxel dephasing for assessment of static thrombus independent of its age (through the presence of intravascular T1-isointense/hyperintense signal or contrast enhancement). In addition, 3D isotropic BBMRI facilitates multiplanar and curved reconstructions to better evaluate the tortuous intracranial arteries. ${ }^{8}$ Our study aimed to assess the accuracy and reliability of BBMRI in diagnosing intracranial large-vessel arterial occlusions.

\section{MATERIALS AND METHODS Patient and Imaging Studies}

Institutional review board approval at Northwestern University was obtained for a retrospective medical record and PACS imaging review of patients with suspected diagnosis of stroke and intracranial thromboembolic occlusions. From October 2014 to June 2018, we identified 199 BBMRI studies and included patients with documented large-vessel arterial occlusions on CTA and/or DSA, performed within 30 days of the BBMRI study. All largevessel arterial occlusions reported on CTA and/or DSA were reviewed and confirmed by a separate interventional neuroradiologist, and whenever discrepancy was found between CTA and DSA, DSA was used as the reference standard. Patients were excluded if they received any intervention (IV/IA tPA thrombolysis and/or mechanical thrombectomy) between BBMRI and reference CTA/ DSA. Imaging exclusion criteria were notable for patient motion artifacts rendering the study nondiagnostic, limited BBMRI scan volumes excluding the ROI/vessel occlusion site, or imaging without the availability of both pre- and post-contrast-enhanced T1 SPACE sequences.

We studied patient demographics, clinical presentations, vessel occlusion sites, ischemic strokes, and age of ischemic strokes using sequence-specific MR imaging findings (DWI, ADC, FLAIR, and unenhanced T1- and T2-weighted images) to classify strokes in the affected arterial territory as acute/subacute or chronic. ${ }^{9}$ Arterial occlusions were classified as acute/subacute $(<3$ weeks) if the patient presented with acute/subacute infarcts in the downstream vascular territory. Chronic/incidental $(>3$ weeks) occlusions were defined if there were no infarcts or chronic infarcts in the downstream vascular territory, the patient presented with acute/subacute infarcts in a different vascular territory, or if previous angiographic studies ( $>3$ weeks before BBMRI) reported an occlusion.

\section{BBMRI Protocol}

BBMRI sequences were acquired on a $3 \mathrm{~T}$ MR imaging scanner (Magnetom Skyra; Siemens) in a clinical setup with a 20-channel head coil. In all cases, a standardized MR imaging stroke protocol was performed, including axial T1 spin-echo, T2 fast spin-echo, DWI, FLAIR, gradient recalled-echo or SWI sequences, TOFMRA, and DSC brain perfusion imaging. Additionally, 3D variable flip angle TSE BBMRI (T1 SPACE) imaging was performed with the following parameters: FOV $=165 \mathrm{~mm}, \mathrm{TR} / \mathrm{TE}=800 / 22$ $\mathrm{ms}$, integrated parallel acquisition technique factor $=2$, number in $\mathrm{z}$-direction $=104$ slices, bandwidth $=372 \mathrm{~Hz} /$ pixel, turbo factor $=30$, scan time $=\sim 8$ minutes, imaging matrix $=320 \times$ 352 , voxel volume $=0.5 \times 0.5 \times 0.6 \mathrm{~mm}$. A single-dose $(1 \mathrm{mmol} /$ $\mathrm{mL}$ ) of gadobutrol, a gadolinium-based T1-shortening contrast agent (Gadavist; Bayer Schering Pharma, Berlin, Germany), was injected, and the scan was repeated 9 minutes after contrast administration. Radial $k$-space view ordering was used to optimize T1-weighted contrast. Images were generated and transferred to a PACS for direct visualization on the workstation.

\section{BBMRI Analysis}

BBMRI (T1 SPACE) images were separately evaluated by 2 independent neuroradiologists on a de-identified PACS workstation. Both observers were blinded to patient demographics, imaging indications, other MR imaging sequences, and other angiographic studies (MRA/CTA/DSA). Qualitative image analyses of the intraluminal arterial hyperintensity and contrast enhancement were performed on the PACS workstation. Intraluminal T1 hyperintensity was graded on a 3-point scale: 0 , none; 1 , weak visualization/hyperintense signal less than that of the intraorbital fat; or 2, strong visualization/hyperintense signal equal to or more than that of the intraorbital fat. Intraluminal enhancement was graded on a 3-point scale: 0 , none or signal equal to that of the pre-contrast image; 1 , weak enhancement with signal greater than that of the pre-contrast image, but less than that of the pituitary stalk; 2, strong enhancement with signal greater than that of the pre-contrast image, equal to or greater than that of the pituitary stalk.

Normal vessels were defined as exhibiting preserved intraluminal black-blood properties with no intraluminal signal intensity or enhancement (Fig 1). Arterial occlusion was defined as a focal area of intraluminal T1-hyperintense signal or enhancement graded as 1 or 2, completely replacing the normal intraluminal black-blood signal on nonenhanced and/or contrast-enhanced T1 SPACE images, respectively (Figs 2 and 3). Both readers were asked to assess a total of 19 intracranial arterial segments in the anterior circulation, including the bilateral supraclinoid internal carotid arteries (ICA); middle cerebral artery (MCA) M1-M2 
(superior/inferior) segments; anterior cerebral artery (ACA) A1-A2 segments; and the posterior circulation, including the posterior cerebral artery (PCA) P1 segments, or posterior communicating arteries if more dominant; $\mathrm{P} 2$ segments; the vertebral artery (VA) V4 segments; and the basilar artery (BA). Aplastic vessel segments not visualized on CTA or DSA imaging were excluded from BBMRI analysis.

\section{Statistical Analysis}

The diagnostic performance of BBMRI was expressed as sensitivity and specificity of intraluminal T1 hyperintensity or contrast enhancement in diagnosing arterial occlusions versus controls, which were defined as normal major intracranial segments by reference standard angiographic studies (CTA and/or DSA). Interobserver agreement for BBMRI intraluminal arterial hyperintensity and contrast enhancement was assessed using the Cohen $\kappa$ coefficient. The Cohen $\kappa$ coefficient varies between 0 and 1 , with 0 representing no agreement and 1 representing complete agreement. $\kappa$ values of $>0.8,>0.6-0.8,>0.4-0.6$, and $\leq 0.4$ indicated excellent, good, fair, and poor agreement, respectively. Subgroup analyses comparing anterior and posterior circulations and acute/ subacute versus chronic/incidental occlusions were performed using Mann-Whitney and Fisher exact tests for continuous and categorical variables, respectively. In case of disagreement, images were reviewed by a third neuroradiologist for consensus grading

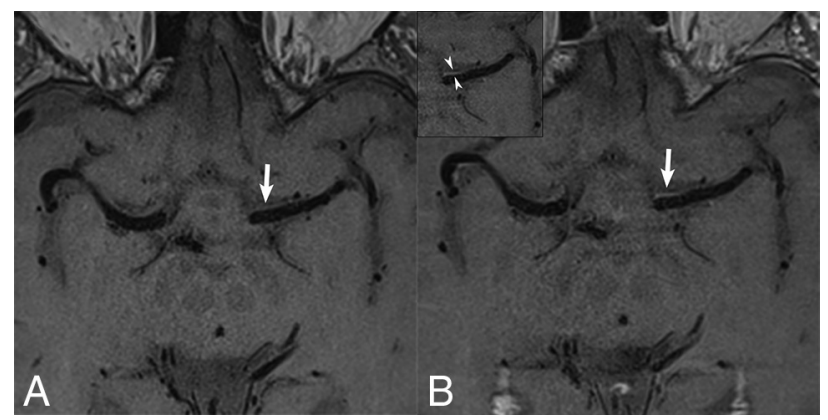

FIG 1. High-resolution axial pre- $(A)$ and post contrast (B) BBMRI demonstrates patent bilateral MCAs with normal intraluminal hypointensity (black-blood) due to flow-suppression techniques. Normal thin vessel wall or marginal periarterial enhancement (arrows) is observed on post-contrast versus pre-contrast imaging, best delineated in the inset (arrowheads).

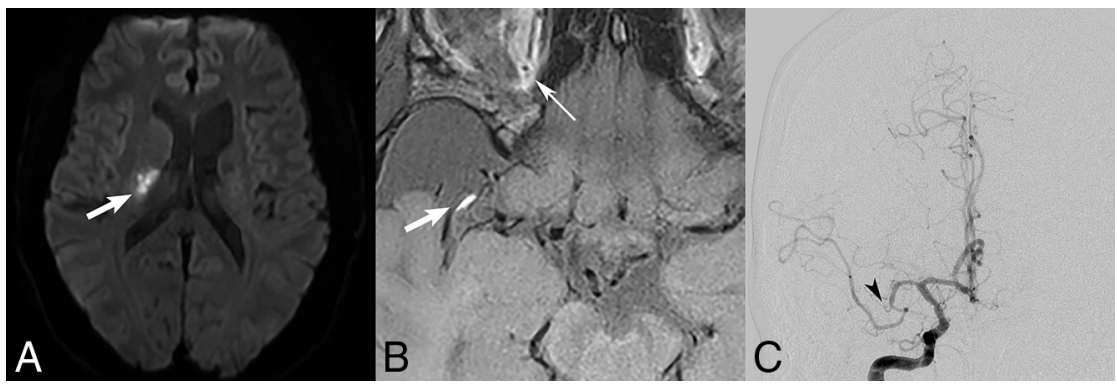

FIG 2. A 39-year-old patient who presented with left-sided hemiplegia. MR DWI axial (A) shows restricted diffusion (arrow) in the periventricular white matter, consistent with an acute infarct. Axial pre-contrast (B) BBMRI demonstrates a focus of grade 2 /strong intraluminal T1 hyperintensity (thick arrow) in the distal M1 segment proximal to the MCA bifurcation, compared with the normal retroglobal orbital fat (thin arrow), suspicious for an acute/subacute occlusion. DSA anteroposterior image $(C)$ confirms the M1 right MCA occlusion (black arrowhead), after supply to a prominent anterior temporal branch. of intraluminal $\mathrm{T} 1$ hyperintensity and contrast enhancement, and utilized for subgroup analyses. A 2-tailed $P$ value $<.05$ was considered statistically significant. Statistical analysis was performed using SPSS statistical software (Version 23.0; IBM, Armonk, New York).

\section{RESULTS}

Thirty-one patients (14 male [45\%], 17 female [55\%]; mean age, 62 years; range, 34-79 years) presented with ischemic stroke symptoms and 38 intracranial large-vessel arterial occlusions that were identified and included in the study. The median time between the reference scan (CTA/DSA) confirming large-vessel occlusions and the BBMRI scan was 2 days (range, 0-20 days). A total of 571/589 major intracranial vessel segments in 31 patients were included in the study ( 6 aplastic segments and 12 normal segments not visualized in the limited BBMRI scan volumes were excluded).

A single arterial occlusion was present in 25/31 patients, 2 occlusions in 5/31 patients, and 3 occlusions in a single patient, providing 38 occlusions and 533 patent segments for independent evaluation by the blinded observers. Arterial occlusions were distributed relatively equally between the anterior and posterior intracranial circulations (58\% versus $42 \%)$ : ACA $(n=4)$ and MCA $(n=18)$ versus the PCA $(n=4)$, VA $(n=10)$, and BA $(n=2)$. Acute/subacute DWI infarcts were observed in 22 patients with 29 occlusions, but only downstream in the vascular distributions of $16 / 29$ occlusions characterized as acute/subacute occlusions, and the remaining 13 were considered chronic/incidental occlusions. Chronic infarcts were noted on T1/T2/FLAIR-weighted images downstream of 3 occlusions in 3 patients, and no infarcts were seen in 6 patients with 6 occlusions, providing a total of 16 acute/ subacute occlusions and 22 chronic/incidental occlusions for analysis.

Both observers successfully identified all intracranial largevessel arterial occlusions on BBMRI by either weak (grade 1) or strong (grade 2) visualization of intraluminal T1 hyperintensity and/or contrast enhancement with no false-negative results (Online Table). Only a single case of a false-positive basilar artery occlusion was reported by both observers on pre- and post-contrast BBMRI and confirmed by CTA, yet deemed to be a severe, long-segment atherosclerotic stenosis with slow stringlike flow rather than an occlusion on DSA. By combining grades 1 and 2 for intraluminal T1 hyperintensity and/or contrast enhancement, we observed excellent sensitivity (100\%) and specificity (99.8\%) with either pre- or postcontrast-enhanced BBMRI, providing near-equivalent diagnostic power and accuracy in the diagnosis of intracranial large-vessel arterial occlusions in comparison with the reference standards of CTA and DSA.

Good interobserver agreement for intraluminal $\mathrm{T} 1$ hyperintensity (unweighted $\kappa=0.63$ ) and excellent interobserver agreement for intraluminal 


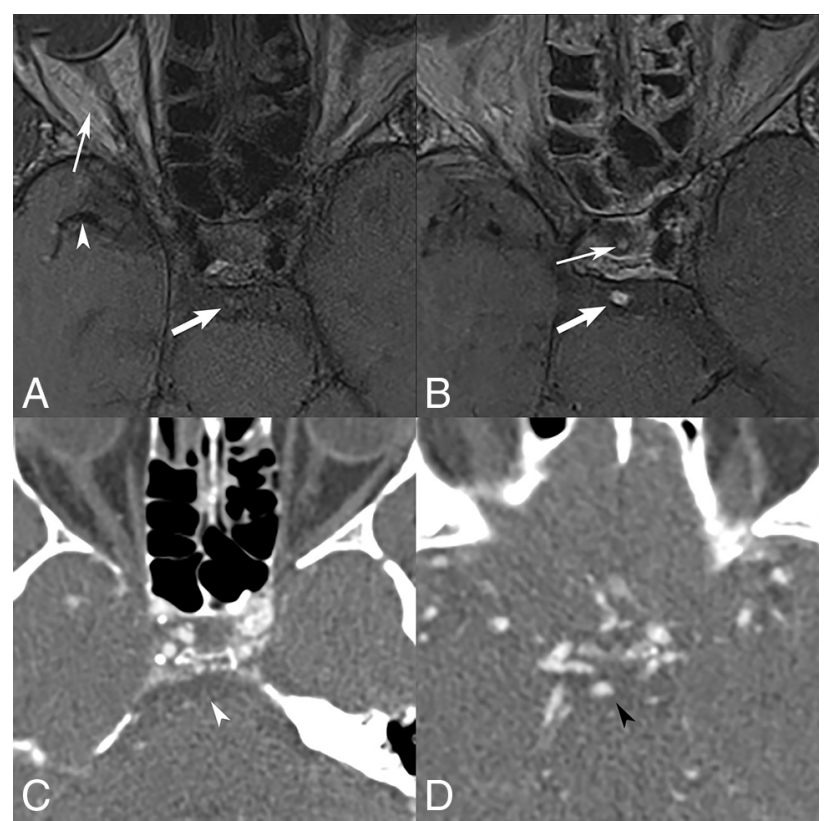

FIG 3. A 63-year-old patient who presented with nausea, vomiting, and double vision secondary to multiple posterior circulation strokes. Axial pre-contrast BBMRI $(A)$ shows a focus of grade $1 /$ weak intraluminal $\mathrm{Tl}$ hyperintensity in the basilar artery (thick arrow), replacing the normal intra-arterial black-blood signal of the M1 segment (arrowhead), but less intense than in the orbital fat (thin arrow). Axial post-contrast BBMRI (B) shows grade 2/strong intraluminal contrast enhancement (thick arrow) of the basilar artery thrombus, compared with the normal avidly enhancing signal of the infundibular stalk (thin arrow). CTA (C and D) at the level of pons and the circle of Willis confirms occlusion of the basilar artery (white arrowhead) with distal basilar tip reconstitution (black arrowhead) from the anterior circulation via the posterior communicating artery.

contrast enhancement (unweighted $\kappa=0.89$ ) were noted, consistent with reliable BBMRI findings in the diagnosis of intraluminal arterial signal abnormality and enhancement. In fact, there was near complete agreement between observers on the presence of strong (grade 2) intraluminal enhancement in 33/38 (87\%) confirmed arterial occlusions with disagreement of grade 1 versus grade 2 enhancement in only a single segment.

On subgroup analyses of anterior versus posterior circulation occlusions (Table 1) and acute/subacute versus chronic/incidental occlusions (Table 2), there were no statistical differences in baseline demographics or time between BBMRI and reference scans. Posterior circulation occlusions had a higher percentage of chronic/incidental occlusions than anterior circulation occlusions $(81.2 \%$ versus $40.9 \%, P=.02)$. Both anterior and posterior circulation occlusions maintained a high degree of contrast enhancement ( $>80 \%$ of occlusions with strong grade 2 enhancement), relatively lower degrees of $\mathrm{T} 1$ hyperintensity $(22.7 \%$ and $43.7 \%$ with strong grade 2 hyperintensity, respectively), and no statistical differences in BBMRI findings between the groups.

Chronic/incidental occlusions had a predilection for the posterior circulation $(59.1 \%)$, whereas most acute/subacute occlusions $(81.2 \%)$ were identified in the anterior circulation $(P=.02)$. Both acute/subacute and chronic/incidental occlusions continued to demonstrate a high degree of contrast enhancement ( $>80 \%$ of occlusions with a strong grade 2 enhancement) and no statistical differences. However, chronic/incidental occlu-
Table 1: Subgroup analysis of anterior versus posterior circulation occlusions

\begin{tabular}{|c|c|c|c|}
\hline & $\begin{array}{l}\text { Anterior } \\
(n=22)\end{array}$ & $\begin{array}{l}\text { Posterior } \\
(n=16)\end{array}$ & $\begin{array}{c}P \\
\text { Value }\end{array}$ \\
\hline Mean age (range) (yr) & $59.3(34-77)$ & $63.1(39-79)$ & .24 \\
\hline Male (\%) & 40.9 & 62.5 & .32 \\
\hline $\begin{array}{l}\text { Median time between BBMRI and } \\
\text { CTA/DSA days (range) (day) }\end{array}$ & $3(0-18)$ & $1(0-20)$ & .62 \\
\hline Occlusion chronicity (\%) & & & .02 \\
\hline Acute/subacute & 59.1 & 18.8 & \\
\hline Chronic/incidental & 40.9 & 81.2 & \\
\hline Hyperintensity (\%) & & & .29 \\
\hline Grade 1 & 77.3 & 56.2 & \\
\hline Grade 2 & 22.7 & 43.7 & \\
\hline Enhancement (\%) & & & .99 \\
\hline Grade 1 & 18.2 & 12.5 & \\
\hline Grade 2 & 81.8 & 87.5 & \\
\hline
\end{tabular}

Table 2: Subgroup analysis of acute/subacute versus chronic/ incidental occlusions

\begin{tabular}{lccc}
\hline & $\begin{array}{c}\text { Acute/ } \\
\text { Subacute } \\
(\boldsymbol{n}=16)\end{array}$ & $\begin{array}{c}\text { Chronic/ } \\
\text { Incidental } \\
(\boldsymbol{n}=22)\end{array}$ & $\begin{array}{c}\boldsymbol{P} \\
\text { Value }\end{array}$ \\
\hline Mean age (range) (yr) & $58.1(34-77)$ & $62.9(37-79)$ & .16 \\
Male (\%) & $50 \%$ & $50 \%$ & .99 \\
Median time between BBMRI & $3(0-18)$ & $1(0-20)$ & .8 \\
$\quad$ and CTA/DSA (range) (day) & & & \\
Occlusion site (\%) & & & $.02^{\mathrm{a}}$ \\
$\quad$ Anterior & 81.2 & 40.9 & \\
$\quad$ Posterior & 18.8 & 59.1 & \\
Hyperintensity (\%) & & & $.04^{\mathrm{a}}$ \\
$\quad$ Grade 1 & & & \\
$\quad$ Grade 2 & 87.5 & 54.5 & \\
Enhancement (\%) & 12.5 & 45.5 & \\
$\quad \begin{array}{l}\text { Grade 1 } \\
\text { Grade 2 }\end{array}$ & 12.5 & 18.2 & \\
\hline a Significant. & 87.5 & 81.8 & \\
\hline
\end{tabular}

sions exhibited statistically higher grades of T1 hyperintensity on pre-contrast BBMRI compared with acute/subacute occlusions ( $45.5 \%$ versus $12.5 \%$ with strong grade 2 hyperintensity, $P=.04)$.

\section{DISCUSSION}

High-resolution BBMRI or VWI is increasingly used in clinical practice for the evaluation of intracranial vessel wall pathology. ${ }^{8,10}$ Multiple studies have described VWI findings in various intracranial steno-occlusive diseases, including accuracy and reliability measurements in direct comparison with conventional vascular imaging techniques (CTA, MRA, and DSA). ${ }^{10-15}$ BBMRI has been shown to characterize intracranial stenoses with high sensitivity (89\%-93\%) and specificity (53\%-98\%) and excellent interobserver agreement, ${ }^{10,14,15}$ but there have been few independent studies of BBMRI findings to diagnose or differentiate largevessel arterial occlusions.

Hui et $\mathrm{al}^{12}$ reported 10 patients with DSA-confirmed acute/ subacute large-vessel occlusions who underwent BBMRI, but they emphasized vessel wall enhancement patterns rather than intraluminal thrombus characteristics for insight into underlying pathologies. Recently, Bai et al ${ }^{14}$ evaluated 60 patients with 80 suspected MCA intracranial atherosclerotic disease lesions with TOF-MRA, BBMRI, and CTA as the reference standard identifying 15 arterial occlusions. In this small subset, they also noted a 
very high sensitivity (100\%) and specificity (95\%-97\%) in detecting occlusions with VWI, using either source or minimum-intensity black-blood luminal angiography techniques, whereas TOFMRA overestimated the pathology with significantly lower specificity ( $85 \%)$. In a larger series of 62 patients with acute stroke with MR imaging negative for the susceptibility vessel sign and BBMRI, Baik et $\mathrm{al}^{13}$ reported contrast-enhancement patterns of intracranial stenoses and occlusions, identifying strong, round, and concentric enhancement in 15/20 (75\%) patients with suspected acute arterial occlusions compared with $4.7 \%$ with severe intracranial stenoses. These findings correlate well with the $87.5 \%$ strong grade 2 enhancement of acute/subacute occlusions seen in our study. In a separate study, Kim et $\mathrm{al}^{16}$ also studied 20 chronic unilateral MCA occlusions with high-resolution BBMRI and described 2 distinct "plugged" or "vanishing" patterns as either heterogeneous intraluminal signal in 13/20 patients or an absence of vascular structures in 7/20 patients, with shrunken MCA or collateral vessels, respectively. These latter findings suggest a stenoocclusive vasculopathy/Moyamoya pathology rather than thromboembolic occlusions, but the authors did not comment on intraluminal hyperintensity or contrast-enhancement patterns.

Prior literature has suggested that acute, symptomatic, intracranial atherosclerotic disease plaques may be associated with higher degrees of eccentric (extraluminal) contrast enhancement compared with chronic or asymptomatic plaques. ${ }^{17}$ In contrast, we noted persisting strong intraluminal enhancement of chronic/ incidental arterial occlusions, hypothesized to be secondary to gadolinium leakage or superimposed inflammation of organized fibrin and platelet-rich thrombus. Methemoglobin is produced in the early stages ( $>7$ days) of thrombus formation and requires time for accumulation. This feature may account for the lower grades of intraluminal T1 hyperintensity in acute/subacute occlusions that presented with early subacute infarcts ( $<7$ days). Methemoglobin also has the strongest paramagnetic properties for shortening $\mathrm{T} 1$ relaxation times and can persist for several weeks to months, ${ }^{18}$ potentially explaining the statistically higher grades of intraluminal $\mathrm{T} 1$ hyperintensity seen in chronic/incidental occlusions. In fact, 4 patients in our cohort underwent 3-, 6-, 9-, or 18-month follow-up BBMRI scans (data not shown), respectively, with persisting $\mathrm{T} 1$ hyperintensity on precontrast BBMRI in all 4 patients and strong grade 2 enhancement on postcontrast BBMRI in 3/4 patients. Another mechanism for chronic intraluminal T1 hyperintensity may be related to slow flow adjacent to the arterial occlusion site, resulting in impaired intravoxel dispersion and loss of the normal black-blood signal, independent of thrombus chronicity. Although we observed a higher percentage of posterior circulation occlusions diagnosed as chronic/incidental occlusions, this may be explained by the redundant supply of bilateral vertebral arteries with a predilection for silent occlusions (no vertebral artery occlusions presented with acute infarcts on DWI).

In our study, we independently evaluated the accuracy of both pre- and post-contrast BBMRI in diagnosing acute/subacute and chronic/incidental arterial occlusions in all major cerebral arteries, regardless of the underlying etiology, and in a large patient cohort. BBMRI identifies intracranial arterial occlusions with excellent sensitivity and specificity $(100 \%$ and
99.8\%, respectively) compared with CTA and DSA. High accuracy was sustained across anterior versus posterior circulation locations and acute/subacute versus chronic/incidental occlusions. Although there was higher interobserver agreement with $>80 \%$ strong grade 2 intraluminal enhancement on post-contrast BBMRI, all occlusions were also successfully identified as grade 1 or 2 intraluminal T1 hyperintensity, consistent with equal and independent accuracy on pre-contrast BBMRI. These findings suggest that even noncontrast BBMRI may be superior to 3D-TOF-MRA sequences, which have a lower reported sensitivity $(88 \%-100 \%)$ and specificity $(85 \%-$ $97 \%$ ) for detecting intracranial occlusions in comparison with the criterion standard CTA and DSA modalities. ${ }^{15,19}$

Our study had the limitations of a retrospective analysis with its inherent challenges. There could have been inadvertent observer recall bias from prior clinical experience, though we attempted to mitigate this by blinding the observers to patient names and clinical history, allowing significant time intervals since MR imaging, and assessing their agreement. Our classification of arterial occlusion chronicity has the potential for error; therefore, we used an objective paradigm based on their clinical history, MR imaging findings, and prior angiographic studies. The sample size was small, yet to our knowledge, this is the largest series specifically focusing on the utility of BBMRI in the detection of intracranial large-vessel arterial occlusions. Finally, CTA was used as the reference standard when DSA was not available; however, studies have shown $100 \%$ sensitivity and specificity for CTA in detecting largevessel arterial occlusions. ${ }^{20}$

\section{CONCLUSIONS}

3D high-resolution BBMRI provides an adjunctive MR imagingbased mechanism to confidently diagnose large-vessel arterial occlusions in the setting of conventional low-resolution MR imaging and nonspecific MRA findings. BBMRI with or without contrast provides high accuracy in the diagnosis of acute/subacute as well as chronic/incidental occlusions, with near-equivalent sensitivity and specificity to CTA and DSA.

\section{ACKNOWLEDGMENTS}

We thank Saadia Sattar, MSc, who contributed to the statistical analysis of this study.

Disclosures: Timothy J. Carroll—RELATED: Grant: National Institutes of Health, Comments: National Institutes of Health/National Institute of Neurological Disorders and Stroke 1R01NS093908*; UNRELATED: Grants/Grants Pending: National Institutes of Health. * Sameer A. Ansari-RELATED: Grant: American Heart Association 13GRNT17340018 and 14GRNT20380798 (Principal Investigators: S.A. Ansari and T.J. Carroll); National Institutes of Health/National Heart, Lung, and Blood Institute 1R21HL130969 (Principal Investigator: S.A. Ansari); and National Institutes of Health/ National Institute of Neurological Disorders and Stroke 1R01NS093908 (Principal Investigator: T.J. Carroll).* *Money paid to the institution.

\section{REFERENCES}

1. Goyal M, Menon BK, van Zwam WH, et al; HERMES collaborators. Endovascular thrombectomy after large-vessel ischaemic stroke: a meta-analysis of individual patient data from five randomised trials. Lancet 2016;387:1723-31 CrossRef Medline

2. Ciesienski KL, Caravan P. Molecular MRI of thrombosis. Curr Cardiovasc Imaging Rep 2010;4:77-84 Medline 
3. Korogi Y, Takahashi M, Mabuchi N, et al. Intracranial vascular stenosis and occlusion: diagnostic accuracy of three-dimensional, Fourier transform, time-of-flight MR angiography. Radiology 1994; 193:187-93 CrossRef Medline

4. Ishimaru H, Ochi M, Morikawa M, et al. Accuracy of pre- and postcontrast 3D time-of-flight MR angiography in patients with acute ischemic stroke: correlation with catheter angiography. AJNR Am J Neuroradiol 2007;28:923-26 Medline

5. Axel L. Blood flow effects in magnetic resonance imaging. Magn Reson Annu 1986;237-44

6. Stock KW, Wetzel S, Kirsch E, et al. Anatomic evaluation of the circle of Willis: MR angiography versus intraarterial digital subtraction angiography. AJNR Am J Neuroradiol 1996;17:1495-99 Medline

7. Ginat DT, Meyers SP. Intracranial lesions with high signal intensity on T1-weighted MR images: differential diagnosis. Radiographics 2012;32:499-516 CrossRef Medline

8. Mandell DM, Mossa-Basha M, Qiao Y, et al; Vessel Wall Imaging Study Group of the American Society of Neuroradiology. Intracranial vessel wall MRI: principles and expert consensus recommendations of the American Society of Neuroradiology. AJNR Am J Neuroradiol 2017;38:218-29 CrossRef Medline

9. Allen LM, Hasso AN, Handwerker J, et al. Sequence-specific MR imaging findings that are useful in dating ischemic stroke. Radiographics 2012;32: 1285-97 CrossRef Medline

10. Lee NJ, Chung MS, Jung SC, et al. Comparison of high-resolution MR imaging and digital subtraction angiography for the characterization and diagnosis of intracranial artery disease. AJNR Am J Neuroradiol 2016;37:2245-50 CrossRef Medline

11. Kim JM, Jung KH, Sohn $\mathrm{CH}$, et al. High-resolution MR technique can distinguish moyamoya disease from atherosclerotic occlusion. Neurology 2013;80:775-76 CrossRef Medline
12. Hui FK, Zhu X, Jones SE, et al. Early experience in high-resolution MRI for large vessel occlusions. J Neurointerv Surg 2015;7:509-16 CrossRef Medline

13. Baik SH, Kwak HS, Hwang SB, et al. Three-dimensional black blood contrast enhanced magnetic resonance imaging in patients with acute ischemic stroke and negative susceptibility vessel sign. Eur J Radiol 2018;102:188-94 CrossRef Medline

14. Bai X, Lv P, Liu K, et al. 3D black-blood luminal angiography derived from high-resolution $\mathrm{MR}$ vessel wall imaging in detecting MCA stenosis: a preliminary study. AJNR Am J Neuroradiol 2018;39: 1827-32 CrossRef Medline

15. Liu Q, Huang J, Degnan AJ, et al. Comparison of high-resolution MRI with CT angiography and digital subtraction angiography for the evaluation of middle cerebral artery atherosclerotic steno-occlusive disease. Int J Cardiovasc Imaging 2013;29:1491-98 CrossRef Medline

16. Kim SM, Ryu CW, Jahng GH, et al. Two different morphologies of chronic unilateral middle cerebral artery occlusion: evaluation using high-resolution MRI. J Neuroimaging 2014;24:460-66 CrossRef Medline

17. Vakil P, Vranic J, Hurley MC, et al. T1 gadolinium enhancement of intracranial atherosclerotic plaques associated with symptomatic ischemic presentations. AJNR Am J Neuroradiol 2013;34:2252-58 CrossRef Medline

18. Moody AR. Magnetic resonance direct thrombus imaging. J Thromb Haemost 2003;1:1403-09 CrossRef Medline

19. Degnan AJ, Gallagher G, Teng Z, et al. MR angiography and imaging for the evaluation of middle cerebral artery atherosclerotic disease. AJNR Am J Neuroradiol 2012;331427-35 CrossRef Medline

20. Nguyen-Huynh MN, Wintermark M, English J, et al., How accurate is CT angiography in evaluating intracranial atherosclerotic disease? Stroke 2008;39:1184-88 CrossRef Medline 